\title{
Steroidal Molecular Rotors with 1,4-diethynylphenylene Rotators: Experimental and Theoretical Investigations Towards Seeking Efficient Properties
}

Karolina Olszewska ${ }^{1}$, Izabella Jastrzebska ${ }^{2 *}$, Andrzej Łapiński ${ }^{3}$, Marcin Górecki $^{4 *}$, Rosa Santillan ${ }^{5}$, Norberto Farfán ${ }^{6}$, Tomasz Runka ${ }^{1 *}$

${ }^{1}$ Faculty of Materials Engineering and Technical Physics, Poznan University of Technology, Piotrowo 3, 60-965 Poznań, Poland

${ }^{2}$ Faculty of Chemistry, University of Białystok, Ciołkowskiego 1K, 15-254 Białystok, Poland

${ }^{3}$ Institute of Molecular Physics, Polish Academy of Sciences, Smoluchowskiego 17, 60-179 Poznań, Poland

${ }^{4}$ Institute of Organic Chemistry, Polish Academy of Sciences, Kasprzaka 44/52, 01-224 Warsaw, Poland

${ }_{5}^{5}$ Departamento de Química, Centro de Investigación y de Estudios Avanzados del IPN, México D.F. Apdo. Postal 14-740, 07000, Mexico

${ }^{6}$ Facultad de Química, Departamento de Química Orgánica, Universidad Nacional Autónoma de México, 04510 Ciudad de México D.F., Mexico

Supporting Information:

Synthesis S1

Table S1. Results of DFT calculation for a model of rotor 3 S7

Figure S1. ATR-IR spectra of rotors 2, 2D, 3, 3D S14

Figure S2. Polarized spectra of rotors 1, 2 and 2D S15

Figure S3. Polarized Raman spectra of rotors 1D, 3 and 3D after rotating the sample by $90^{\circ}$ S16

Figure S4. The dependencies of intensity vs. angle of rotation of the sample for the band assigned to stretching vibration of $\mathrm{C}=\mathrm{C}$ bonds in the rotator recorded in parallel (XX) polarization for rotor $3 \mathrm{D}$.

Figure S5. An overview of conformational search for compounds 3 carried out at B3LYP/6-31G(d) level of theory

Figure S6. Simulated spectrum of $3 a$ and steroidal unit at

CAM-B3LYP/def2-TZVP/PCM(C $\left(\mathrm{CH}_{3} \mathrm{CN}\right)$ level of theory 


\section{Synthesis}

1,4-Bis(17 $\alpha$-ethynyl-5 $\alpha$-androstane-3 $\beta, 17 \beta$-diacetate)benzene (1) and

\section{1,4-Bis(17 $\alpha$-ethynyl-5 $\alpha$-androstane-3 $\beta, 17 \beta$-diacetate)benzene-d ${ }_{4}$ (1D)}
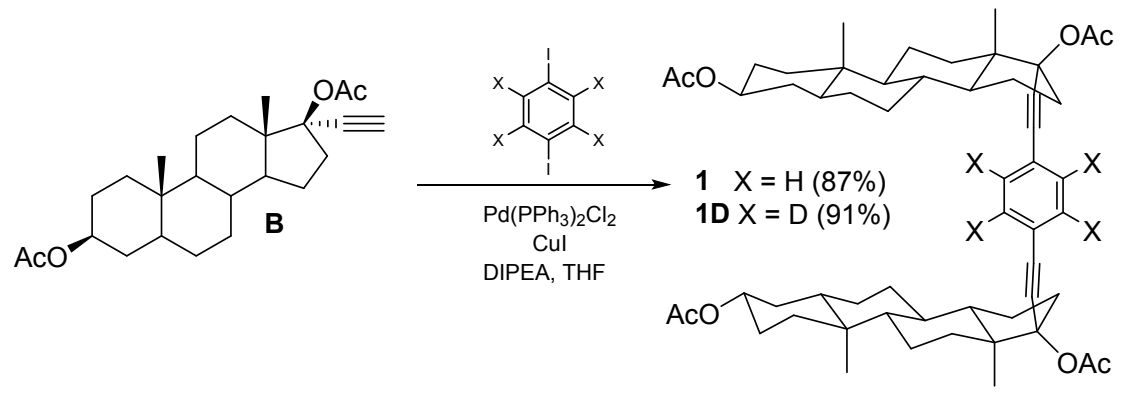

Synthesis of 1: A mixture of $17 \alpha$-Ethynyl-5 $\alpha$-androstane-3 $\beta, 17 \beta$-diacetate(steroid B, $0.2 \mathrm{~g}$, $0.54 \mathrm{mmol})$, 1,4-diiodobenzene (0.09 g, $0.27 \mathrm{mmol}), \mathrm{CuI}(0.01 \mathrm{~g}, 0.05 \mathrm{mmol})$, and $\mathrm{Pd}\left(\mathrm{PPh}_{3}\right)_{2} \mathrm{Cl}_{2}(0.22 \mathrm{~g}, 0.03 \mathrm{mmol})$ in $(i \mathrm{Pr})_{2} \mathrm{NH}(1 \mathrm{ml})$ and $\mathrm{THF}(25 \mathrm{ml})$ was refluxed for 45 min under $\mathrm{N}_{2}$ atmosphere. After this time, the reaction was quenched with saturated $\mathrm{NH}_{4} \mathrm{Cl}$ solution. The organic phase was extracted twice with ethyl acetate and the combined organic portions were dried over anhydrous $\mathrm{Na}_{2} \mathrm{SO}_{4}$. The solvent was removed in vacuo followed by purification (silica gel 70-230) eluting with hexane/ethyl acetate (90/10) affording a product as a white solid $(0.2 \mathrm{~g}, 87 \%)$.

1: M.P. 202-203 ${ }^{\circ}$ C. FTIR-ATR $\left(v, \mathrm{~cm}^{-1}\right):$ 2927, 2866, 2357.2342, 1731, 1230, 1019, 978, 841, 559. ${ }^{1} \mathrm{H}-\mathrm{NMR}\left[500 \mathrm{MHz}, \mathrm{CDCl}_{3}\right](\delta, \mathrm{ppm}) 7.35$ (4H, s, H.-23, H-23'), 4.75-4.60 (1H, m, H-3, H-3'), 2.04 and 2.00 (3Hea, 2s, OAc), 0.88 and 0.83 (3Hea, 2s, Me-18, Me-19). ${ }^{13} \mathrm{C}-$ NMR [125.76 MHz, $\left.\mathrm{CDCl}_{3}\right](\delta, \mathrm{ppm}): 170.8$ and $169.6(2 \mathrm{C}=\mathrm{O}), 131.7(\mathrm{C}-23), 122.9(\mathrm{C} 22)$, 91.0 (C-20), 86.2 and 85.2 (C-21 and C-17), 73.7 (C-3), 53.8 (C-9), 49.1 (C-14), 48.3 (C-13), 44.6 (C-5), 37.5 (C-1), 36.8 (C-10), 36.0 (C-8), 35.6 (C-16), 34.0 and 33.4 (C-12 and C-4), 31.6 (C-7), 28.5 and 27.5, (C-2 and C-6), 23.8 (C-15), 21.6 (OAc), 21.6 (OAc), 20.9 (C-11), 13.8 (C-18), 12.3 (C-19). HRMS (APCI-TOF), calculated for $\left[\mathrm{C}_{56} \mathrm{H}_{74} \mathrm{O}_{8}+\mathrm{H}\right]^{+}$875.5456; Found: 875.5451. Error -0.64 ppm. 
${ }^{1} \mathrm{H}$ NMR spectrum for $\mathbf{1}\left(\mathrm{CDCl}_{3}\right)$

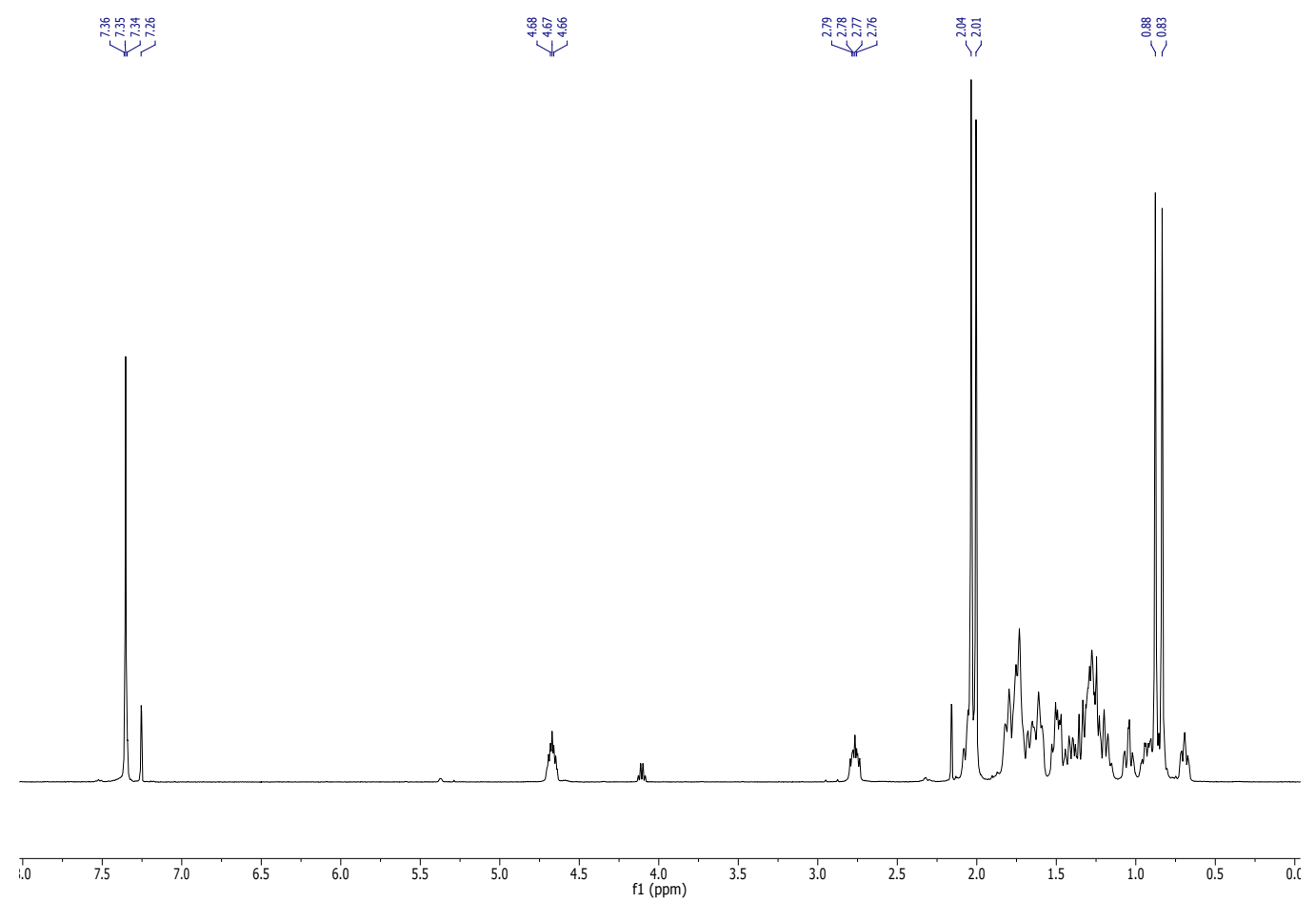

${ }^{13} \mathrm{C}$ NMR spectrum for $\mathbf{1}\left(\mathrm{CDCl}_{3}\right)$

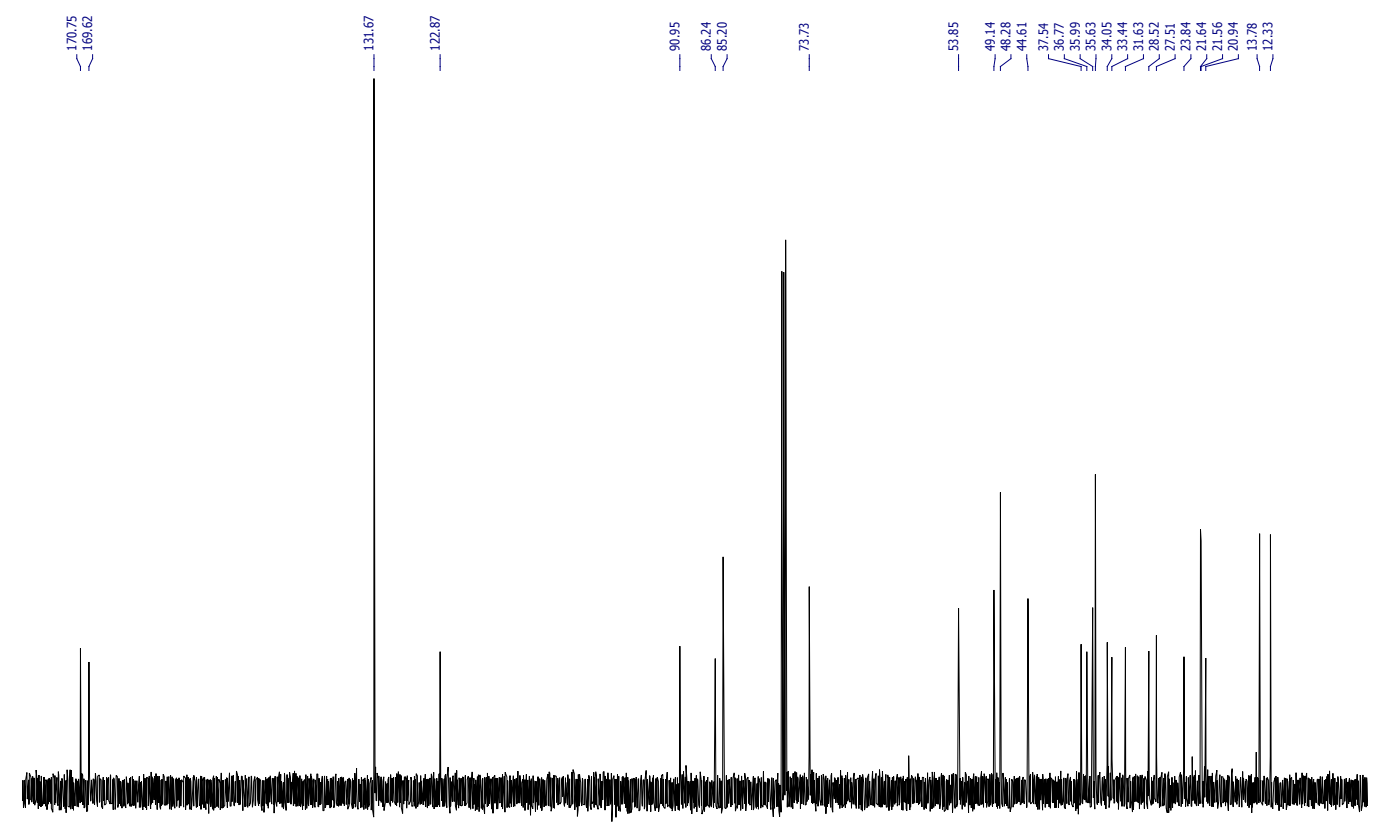


Synthesis of 1D: A mixture of $17 \alpha$-Ethynyl-5 $\alpha$-androstane- $3 \beta, 17 \beta$-diacetate $(0.2 \mathrm{~g}, 0.54$ mmol), 1,4-diiodobenzene- $\mathrm{d}_{4}(0.09 \mathrm{~g}, 0.27 \mathrm{mmol}), \mathrm{CuI}(0.01 \mathrm{~g}, 0.05 \mathrm{mmol})$, and $\mathrm{Pd}\left(\mathrm{PPh}_{3}\right)_{2} \mathrm{Cl}_{2}$ $(0.22 \mathrm{~g}, 0.03 \mathrm{mmol})$ in $(i \operatorname{Pr})_{2} \mathrm{NH}(1 \mathrm{ml})$ and THF $(25 \mathrm{ml})$ was refluxed for $45 \mathrm{~min}$ under $\mathrm{N}_{2}$ atmosphere. After this time, the reaction was quenched with saturated $\mathrm{NH}_{4} \mathrm{Cl}$ solution. The organic phase was extracted twice with ethyl acetate and the combined organic portions were dried over anhydrous $\mathrm{Na}_{2} \mathrm{SO}_{4}$. The solvent was removed in vacuo followed by purification (silica gel 70-230) elutingwith hexane/ethyl acetate (90/10) affording a product as a white solid $(0.21 \mathrm{~g}, 91 \%)$.

1D: M.P.: 196-197 ${ }^{\circ} \mathrm{C} .{ }^{1} \mathrm{H}-\mathrm{NMR}\left[500 \mathrm{MHz}, \mathrm{CDCl}_{3}\right](\delta$, ppm)4.74-4.66 (1H, m, H-3, H-3'), 2.07 and 2.04 (3Hea, 2s, OAc), 0.88 and 0.82 (3Hea, 2s, Me-18, Me-19). ${ }^{13} \mathrm{C}-\mathrm{NMR}$ [125.76

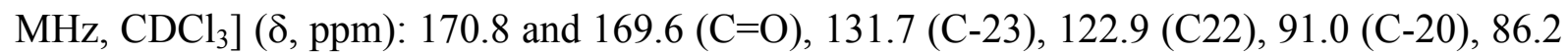
and 85.2 (C-21 and C-17), 73.7 (C-3), 53.8 (C-9), 49.1 (C-14), 48.3 (C-13), 44.6 (C-5), 37.5 (C-1), 36.8 (C-10), 36.0 (C-8), 35.6 (C-16), 34.0 and 33.4 (C-12 and C-4), 31.6 (C-7), 28.5 and 27.5, (C-2 and C-6), 23.8 (C-15), 21.6 (OAc), 21.6 (OAc), 20.9 (C-11), 13.8 (C-18), 12.3 (C-19). HPLC/MS agilent technologies SQ (ESI) calculated for $\left[\mathrm{C}_{56} \mathrm{H}_{70} \mathrm{D}_{4} \mathrm{O}_{8}+\mathrm{H}\right]^{+} 879.6$; Found: 879.6. 
${ }^{1} \mathrm{H}$ NMR spectrum for $\mathbf{1 D}\left(\mathrm{CDCl}_{3}\right)$

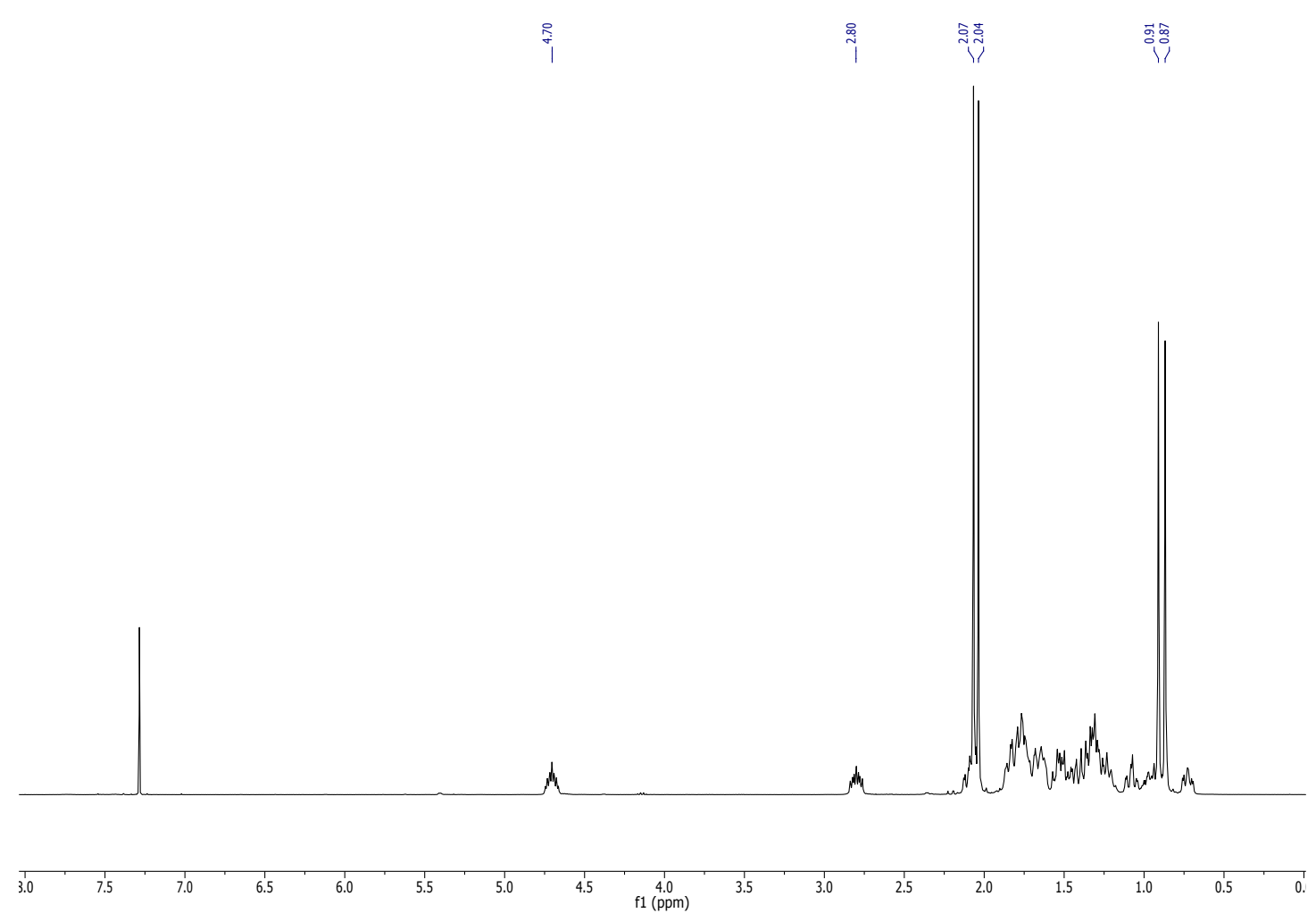

${ }^{13} \mathrm{C} \mathrm{NMR}$ spectrum for $\mathbf{1 D}\left(\mathrm{CDCl}_{3}\right)$

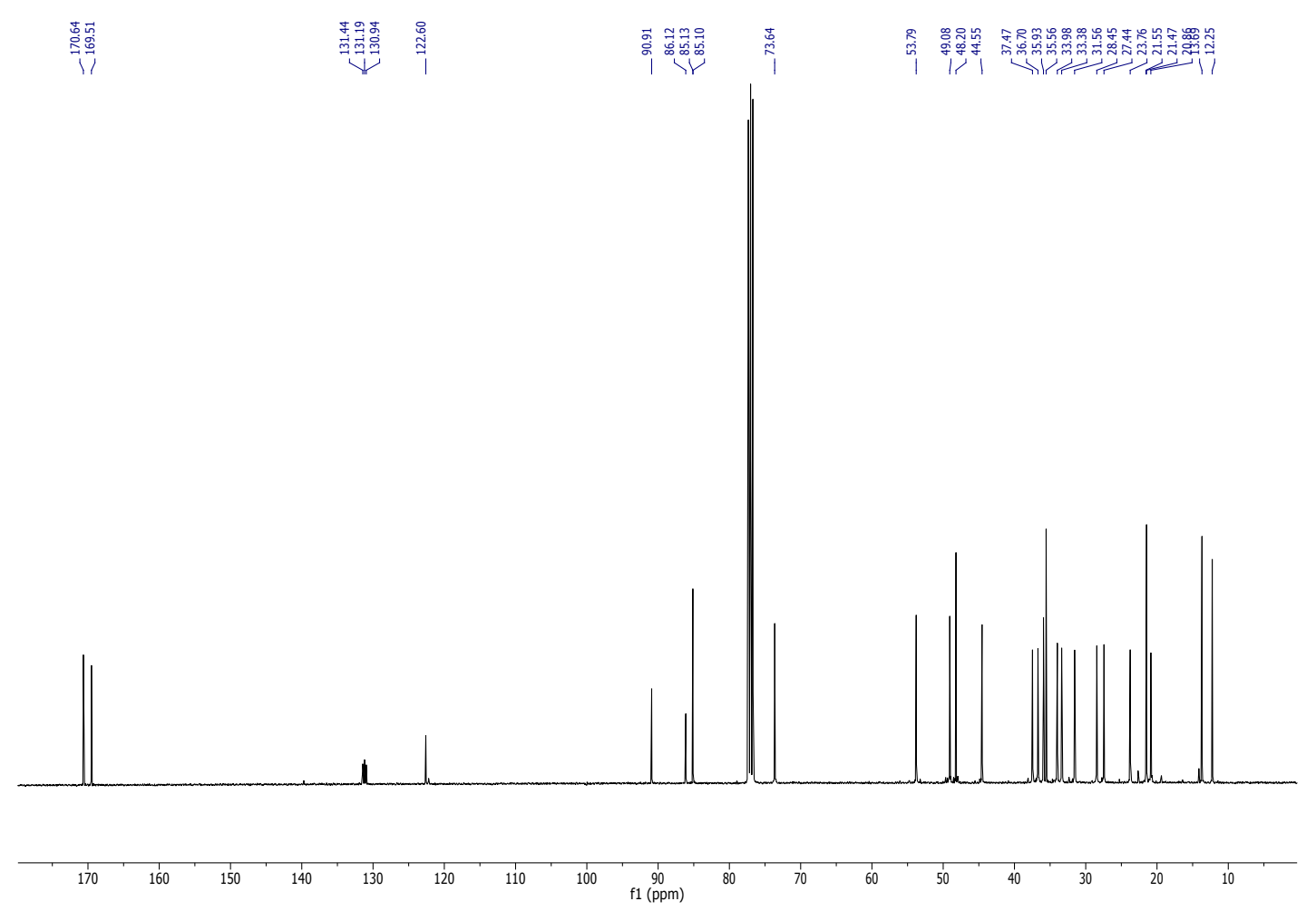




\section{1,4-Bis[4-estren-17 $\alpha$-ethynyl-18a-homo-17ß-ol-3-one]-benzene-d ${ }_{4}(3 D)$}
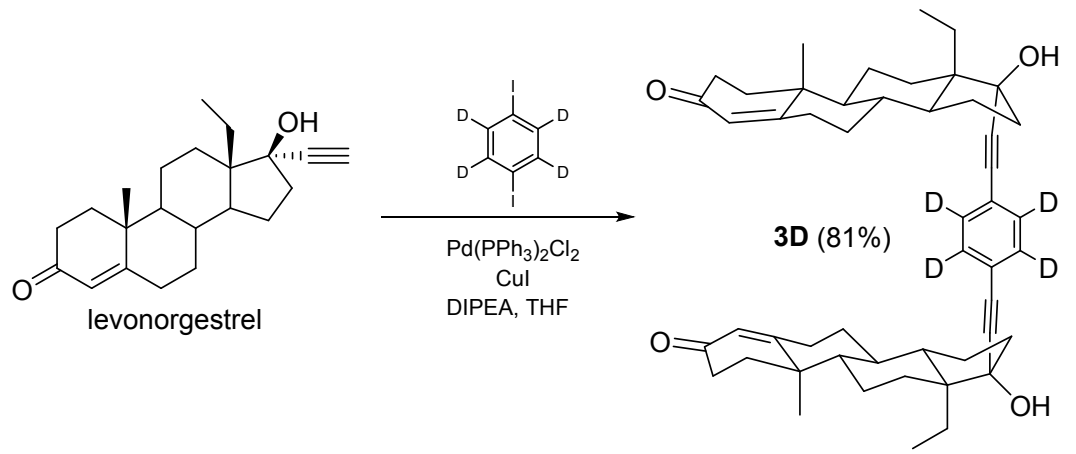

A mixture of levonorgestrel( $0.4 \mathrm{~g}, 1.28 \mathrm{mmol})$, 1,4-diiodobenzene- $\mathrm{d}_{4}(0.21 \mathrm{~g}$, $0.64 \mathrm{mmol}), \mathrm{CuI}(0.024 \mathrm{~g}, 0.128 \mathrm{mmol})$, and $\mathrm{Pd}\left(\mathrm{PPh}_{3}\right)_{2} \mathrm{Cl}_{2}(0.05 \mathrm{~g}, 0.07 \mathrm{mmol})$ in $(i \operatorname{Pr})_{2} \mathrm{NH}(1$ $\mathrm{ml}$ ) and THF $(25 \mathrm{ml})$ was refluxed for $45 \mathrm{~min}$ under $\mathrm{N}_{2}$ atmosphere. After this time, the reaction was quenched with saturated $\mathrm{NH}_{4} \mathrm{Cl}$ solution. The organic phase was extracted twice with ethyl acetate and the combined organic portions were dried over anhydrous $\mathrm{Na}_{2} \mathrm{SO}_{4}$. The solvent was removed under reduced pressure followed by purification (silica gel 70-230) eluting with hexane/ethyl acetate (90/10) affording a product as a crystalline brown solid $(0.37 \mathrm{~g}, 81 \%)$.

3D: M.P. $210-211^{\circ} \mathrm{C} ;{ }^{1} \mathrm{H}$ NMR (500 MHz, $\left.\mathrm{CDCl}_{3}\right) 5.83$ (s, 2H, H4), 1.03 (t, J = $7.4 \mathrm{~Hz}, 6 \mathrm{H}$, C1-8a). ${ }^{13} \mathrm{C}$ NMR (125 MHz, $\left.\mathrm{CDCl}_{3}\right) \delta 200.0$ (C3), 166.6 (C5), 131.2 (C23, t, J = 131.2 Hz), 124.8 (C4), 122.6 (C22), 94.9 (C20), 85.7 (C21), 82.0 (C17), 51.2 (C14), 49.1 (C9), 48.6 (C13), 42.6 (C10), 41.0 (C8), 39.8 (C16), 36.6 (C2), 35.6 (C6), 30.8 (C7), 28.9 (C12), 26.6 (C1), 26.3 (C11), 22.7 (C15), 19.2 (C18), 9.7 (C18a). HRMS (ESI) calculated for $\left[\mathrm{C}_{48} \mathrm{H}_{54} \mathrm{D}_{4} \mathrm{O}_{4}+\mathrm{H}\right]^{+} \mathrm{m} / \mathrm{z}: 703.4720$; found: 703.4721 . 
${ }^{1} \mathrm{H}$ NMR spectrum for $3 \mathrm{D}\left(\mathrm{CDCl}_{3}\right)$
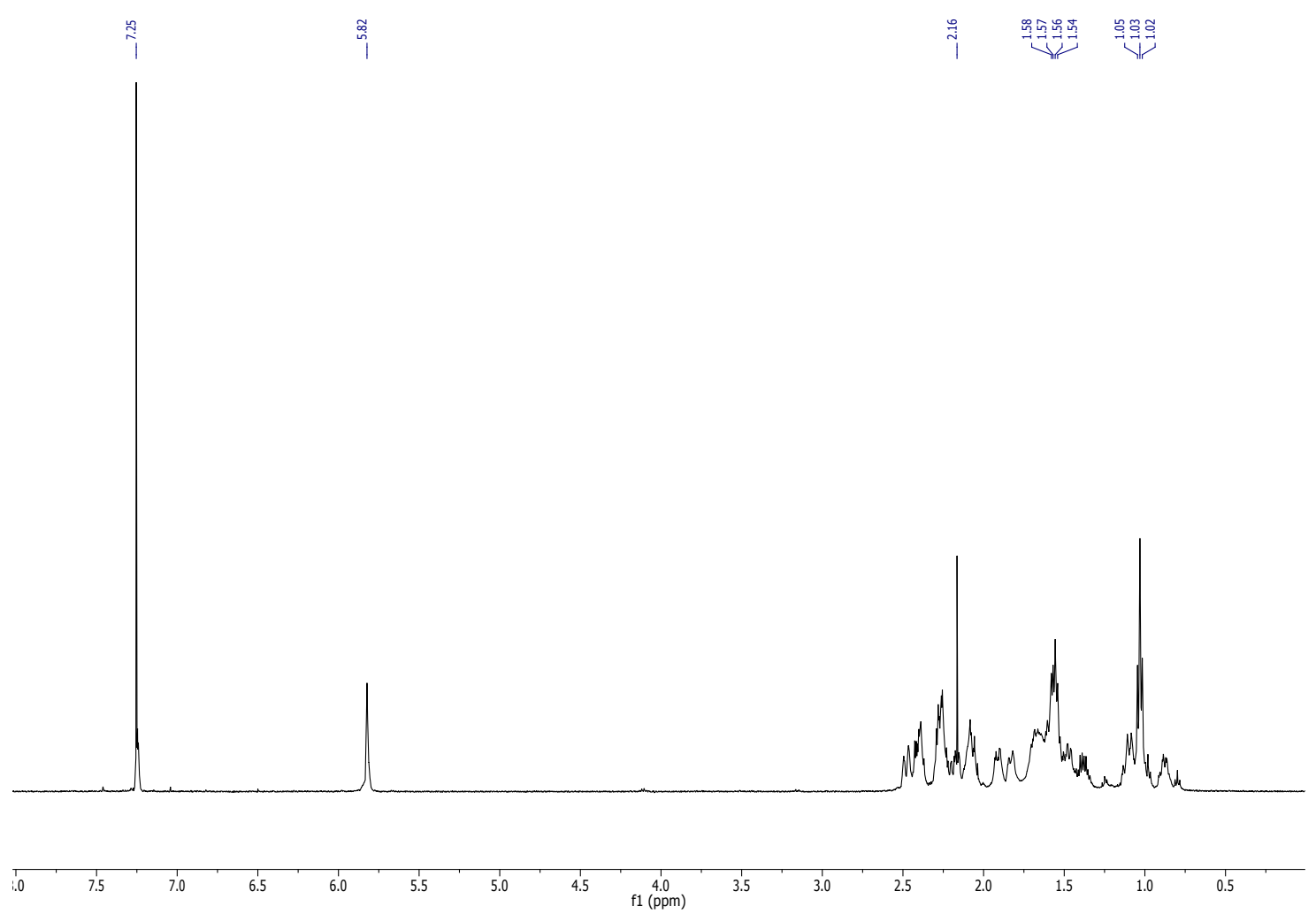

${ }^{13} \mathrm{C} \mathrm{NMR}$ spectrum for $3 \mathrm{D}\left(\mathrm{CDCl}_{3}\right)$

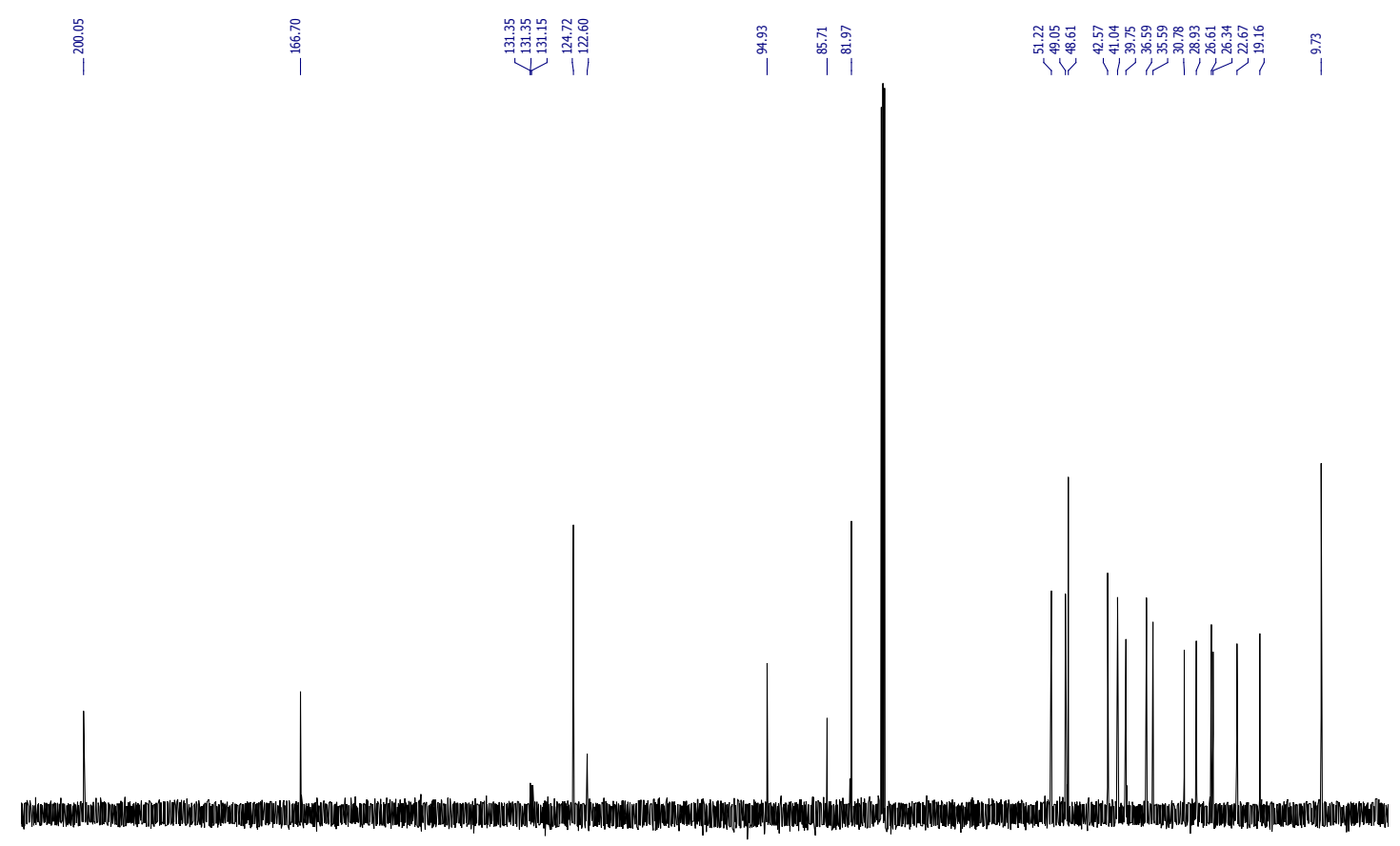

$\begin{array}{llllllllll}200 & 190 & 180 & 170 & 160 & 150 & 140 & 130 & 120 & 110 \\ \mathrm{f} 1(\mathrm{ppm}) & 100\end{array}$ 
Table S1. Results of DFT calculation for a model of rotor 3 in the $100-3500 \mathrm{~cm}^{-1}$ range (wavenumbers were scaled with a factor of 0.961 )

\begin{tabular}{|c|c|c|c|c|}
\hline Lp. & Wavenumber $/ \mathbf{c m}^{-1}$ & $\begin{array}{c}\text { Raman Intensity / } \\
\text { a.u. } \\
\end{array}$ & $\begin{array}{c}\text { IR Intensity / } \\
\text { a.u. } \\
\end{array}$ & Proposed assignment \\
\hline 1 & 103 & 4158 & 0,05 & \multirow[t]{21}{*}{ skeletal vibration } \\
\hline 2 & 105 & 4943 & 0,05 & \\
\hline 3 & 110 & 4148 & 0,22 & \\
\hline 4 & 129 & 2217 & 0,03 & \\
\hline 5 & 138 & 320 & 1,53 & \\
\hline 6 & 145 & 2039 & 0,13 & \\
\hline 7 & 150 & 1693 & 1,39 & \\
\hline 8 & 152 & 671 & 0,86 & \\
\hline 9 & 152 & 289 & 0,75 & \\
\hline 10 & 158 & 141 & 1,59 & \\
\hline 11 & 166 & 1051 & 0,09 & \\
\hline 12 & 172 & 143 & 1,85 & \\
\hline 13 & 180 & 1450 & 0,71 & \\
\hline 14 & 181 & 65 & 2,32 & \\
\hline 15 & 190 & 3385 & 0,56 & \\
\hline 16 & 203 & 6167 & 0,26 & \\
\hline 17 & 208 & 619 & 0,93 & \\
\hline 18 & 217 & 480 & 0,24 & \\
\hline 19 & 220 & 1180 & 1,36 & \\
\hline 20 & 221 & 2963 & 0,53 & \\
\hline 21 & 223 & 2379 & 0,65 & \\
\hline 22 & 252 & 3563 & 15,51 & skeletal vibration $+\delta \mathrm{O}-\mathrm{H}(\mathrm{S})$ \\
\hline 23 & 253 & 3769 & 1,65 & \multirow[t]{3}{*}{ skeletal vibration } \\
\hline 24 & 257 & 46 & 3,14 & \\
\hline 25 & 260 & 965 & 5,97 & \\
\hline 26 & 275 & 1471 & 39,32 & skeletal vibration $+\delta \mathrm{O}-\mathrm{H}(\mathrm{S})$ \\
\hline 27 & 280 & 685 & 48,20 & \multirow[t]{3}{*}{ skeletal vibration } \\
\hline 28 & 280 & 597 & 4,87 & \\
\hline 29 & 290 & 1173 & 3,97 & \\
\hline 30 & 294 & 1131 & 23,78 & skeletal vibration $+\delta \mathrm{O}-\mathrm{H}(\mathrm{S})$ \\
\hline 31 & 301 & 307 & 25,05 & skeletal vibration \\
\hline 32 & 311 & 2661 & 69,02 & \multirow[t]{2}{*}{ skeletal vibration $+\delta \mathrm{O}-\mathrm{H}(\mathrm{S})$} \\
\hline 33 & 323 & 387 & 15,39 & \\
\hline 34 & 334 & 501 & 4,06 & \multirow[t]{3}{*}{ skeletal vibration } \\
\hline 35 & 335 & 936 & 10,00 & \\
\hline 36 & 335 & 181 & 9,08 & \\
\hline 37 & 341 & 709 & 31,57 & skeletal vibration $+\delta \mathrm{O}-\mathrm{H}(\mathrm{S})$ \\
\hline 38 & 358 & 946 & 1,37 & \multirow[t]{6}{*}{ skeletal vibration } \\
\hline 39 & 363 & 1085 & 4,65 & \\
\hline 40 & 381 & 1876 & 0,42 & \\
\hline 41 & 382 & 1115 & 0,76 & \\
\hline 42 & 394 & 109 & 2,94 & \\
\hline 43 & 396 & 245 & 1,59 & \\
\hline
\end{tabular}




\begin{tabular}{|c|c|c|c|c|}
\hline 44 & 396 & 47 & 1,04 & \\
\hline 45 & 406 & 166 & 6,80 & \\
\hline 46 & 415 & 479 & 0,13 & \\
\hline 47 & 424 & 243 & 2,21 & \\
\hline 48 & 425 & 255 & 1,19 & \\
\hline 49 & 446 & 155 & 2,80 & \\
\hline 50 & 448 & 334 & 5,04 & \\
\hline 51 & 453 & 153 & 9,70 & \\
\hline 52 & 458 & 854 & 4,61 & \\
\hline 53 & 464 & 1520 & 1,19 & \\
\hline 54 & 466 & 384 & 0,81 & \\
\hline 55 & 468 & 742 & 3,61 & \\
\hline 56 & 470 & 272 & 5,18 & \\
\hline 57 & 477 & 1507 & 1,93 & \\
\hline 58 & 495 & 961 & 0,96 & \\
\hline 59 & 502 & 1350 & 2,97 & \\
\hline 60 & 509 & 874 & 0,42 & \\
\hline 61 & 510 & 1232 & 5,63 & \\
\hline 62 & 517 & 835 & 0,50 & \\
\hline 63 & 522 & 139 & 3,09 & \\
\hline 64 & 528 & 1857 & 0,13 & \\
\hline 65 & 546 & 96 & 5,46 & \\
\hline 66 & 547 & 47 & 2,08 & \\
\hline 67 & 549 & 135 & 0,17 & \\
\hline 68 & 557 & 3096 & 0,85 & \\
\hline 69 & 587 & 2466 & 6,15 & \\
\hline 70 & 598 & 791 & 0,31 & \\
\hline 71 & 599 & 477 & 0,85 & \\
\hline 72 & 609 & 1175 & 4,72 & \\
\hline 73 & 619 & 838 & 1,31 & \\
\hline 74 & 623 & 468 & 5,80 & \\
\hline 75 & 631 & 537 & 0,50 & \\
\hline 76 & 638 & 402 & 0,02 & v C-C-Casym. (R) \\
\hline 77 & 645 & 333 & 11,89 & \multirow[t]{3}{*}{ v C-C (A) } \\
\hline 78 & 651 & 3023 & 1,27 & \\
\hline 79 & 682 & 413 & 10,42 & \\
\hline 80 & 689 & 641 & 4,47 & \multirow[t]{2}{*}{ v C-C (S) } \\
\hline 81 & 692 & 116 & 10,68 & \\
\hline 82 & 708 & 999 & 0,08 & v C-C-Casym. (R) \\
\hline 83 & 727 & 453 & 2,80 & \multirow[t]{4}{*}{ v C-C (S) } \\
\hline 84 & 741 & 608 & 11,77 & \\
\hline 85 & 744 & 739 & 4,87 & \\
\hline 86 & 746 & 104 & 2,38 & \\
\hline 87 & 764 & 1600 & 3,22 & v C-C-C (R) \\
\hline 88 & 791 & 141 & 1,08 & \multirow[t]{3}{*}{ v C-C (S) } \\
\hline 89 & 794 & 217 & 0,76 & \\
\hline 90 & 798 & 74 & 1,14 & \\
\hline
\end{tabular}




\begin{tabular}{|c|c|c|c|c|}
\hline 91 & 800 & 73 & 1,56 & \\
\hline 92 & 800 & 61 & 0,63 & \\
\hline 93 & 807 & 182 & 1,88 & v C-C-C (R) \\
\hline 94 & 817 & 328 & 0,54 & \multirow[t]{2}{*}{$\gamma \mathrm{C}-\mathrm{H}(\mathrm{R})$} \\
\hline 95 & 823 & 38 & 36,77 & \\
\hline 96 & 826 & 282 & 2,89 & \multirow[t]{7}{*}{ v C-C (S) } \\
\hline 97 & 829 & 328 & 1,75 & \\
\hline 98 & 843 & 165 & 1,43 & \\
\hline 99 & 844 & 84 & 4,20 & \\
\hline 100 & 853 & 202 & 7,24 & \\
\hline 101 & 854 & 48 & 8,85 & \\
\hline 102 & 854 & 440 & 6,76 & \\
\hline 103 & 860 & 1575 & 3,80 & breathing mode (R) \\
\hline 104 & 884 & 58 & 18,47 & \multirow[t]{6}{*}{ v C-C (S) } \\
\hline 105 & 884 & 100 & 9,31 & \\
\hline 106 & 909 & 314 & 6,77 & \\
\hline 107 & 909 & 374 & 7,99 & \\
\hline 108 & 920 & 2854 & 3,69 & \\
\hline 109 & 921 & 314 & 1,31 & \\
\hline 110 & 927 & 6 & 0,01 & \multirow[t]{2}{*}{$\gamma \mathrm{C}-\mathrm{H}(\mathrm{R})$} \\
\hline 111 & 937 & 402 & 0,09 & \\
\hline 112 & 941 & 691 & 3,23 & \multirow[t]{12}{*}{ v C-C (S) } \\
\hline 113 & 942 & 769 & 3,48 & \\
\hline 114 & 948 & 835 & 3,25 & \\
\hline 115 & 949 & 496 & 10,16 & \\
\hline 116 & 951 & 625 & 9,66 & \\
\hline 117 & 952 & 707 & 2,96 & \\
\hline 118 & 964 & 1446 & 7,91 & \\
\hline 119 & 965 & 1180 & 7,03 & \\
\hline 120 & 973 & 1353 & 4,78 & \\
\hline 121 & 974 & 113 & 7,11 & \\
\hline 122 & 989 & 588 & 16,89 & \\
\hline 123 & 990 & 735 & 23,95 & \\
\hline 124 & 997 & 133 & 3,52 & \multirow[t]{3}{*}{$\delta \mathrm{C}$-Hasym. (R) } \\
\hline 125 & 998 & 244 & 0,79 & \\
\hline 126 & 999 & 25 & 2,88 & \\
\hline 127 & 1002 & 418 & 14,42 & \multirow[t]{7}{*}{ v C-C (S) } \\
\hline 128 & 1003 & 240 & 7,02 & \\
\hline 129 & 1018 & 1396 & 6,93 & \\
\hline 130 & 1019 & 250 & 9,00 & \\
\hline 131 & 1024 & 2210 & 8,54 & \\
\hline 132 & 1028 & 244 & 22,29 & \\
\hline 133 & 1030 & 113 & 5,87 & \\
\hline 134 & 1030 & 575 & 10,34 & \multirow[t]{4}{*}{$\mathrm{v}$ C-C (A and S) } \\
\hline 135 & 1034 & 932 & 26,67 & \\
\hline 136 & 1035 & 615 & 29,56 & \\
\hline 137 & 1038 & 183 & 17,81 & \\
\hline
\end{tabular}




\begin{tabular}{|c|c|c|c|c|}
\hline 138 & 1039 & 642 & 6,46 & \multirow[t]{7}{*}{ v C-C (S) } \\
\hline 139 & 1047 & 202 & 10,08 & \\
\hline 140 & 1047 & 478 & 1,33 & \\
\hline 141 & 1061 & 400 & 14,90 & \\
\hline 142 & 1064 & 686 & 2,37 & \\
\hline 143 & 1083 & 740 & 10,06 & \\
\hline 144 & 1086 & 795 & 4,51 & \\
\hline 145 & 1094 & 63 & 7,75 & v C-C $(\mathrm{S})+\tau \mathrm{C}-\mathrm{H}$ in $\mathrm{CH}_{2}$ groups (S) \\
\hline 146 & 1095 & 46 & 3,80 & \multirow[t]{2}{*}{ 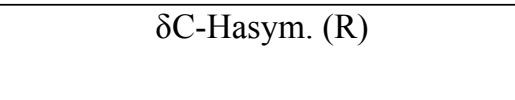 } \\
\hline 147 & 1095 & 20 & 6,91 & \\
\hline 148 & 1113 & 734 & 25,90 & \multirow[t]{11}{*}{ v C-C (S) $+\tau \mathrm{C}-\mathrm{H}$ in $\mathrm{CH}_{2}$ groups (S) } \\
\hline 149 & 1113 & 341 & 11,85 & \\
\hline 150 & 1114 & 189 & 12,19 & \\
\hline 151 & 1116 & 1151 & 12,90 & \\
\hline 152 & 1125 & 236 & 11,77 & \\
\hline 153 & 1127 & 584 & 3,65 & \\
\hline 154 & 1133 & 1968 & 6,51 & \\
\hline 155 & 1134 & 393 & 2,70 & \\
\hline 156 & 1141 & 944 & 5,30 & \\
\hline 157 & 1142 & 1658 & 0,72 & \\
\hline 158 & 1160 & 266 & 5,85 & \\
\hline 159 & 1163 & 5904 & 6,91 & \multirow[t]{2}{*}{$\delta$ C-Hi.ph. (R) } \\
\hline 160 & 1166 & 9435 & 2,02 & \\
\hline 161 & 1172 & 195 & 9,18 & \multirow{6}{*}{ v C-C $(\mathrm{S})+\tau \mathrm{C}-\mathrm{H}$ in $\mathrm{CH}_{2}$ groups $(\mathrm{S})$} \\
\hline 162 & 1172 & 368 & 2,72 & \\
\hline 163 & 1180 & 99 & 16,76 & \\
\hline 164 & 1186 & 915 & 12,80 & \\
\hline 165 & 1194 & 653 & 49,65 & \\
\hline 166 & 1195 & 515 & 52,04 & \\
\hline 167 & 1202 & 562 & 7,35 & \multirow[t]{4}{*}{$\tau \mathrm{C}-\mathrm{H}$ in $\mathrm{CH}_{2}$ groups (S) } \\
\hline 168 & 1207 & 2540 & 1,79 & \\
\hline 169 & 1215 & 1506 & 13,58 & \\
\hline 170 & 1218 & 347 & 5,80 & \\
\hline 171 & 1222 & 421 & 7,03 & \multirow[t]{2}{*}{$\tau \mathrm{C}-\mathrm{H}$ in $\mathrm{CH}$ groups $(\mathrm{S})$} \\
\hline 172 & 1222 & 227 & 9,84 & \\
\hline 173 & 1228 & 9201 & 23,68 & $\begin{array}{c}\text { v C-C (A) and } \tau \mathrm{C}-\mathrm{H} \text { in } \mathrm{CH}_{2} \text { groups } \\
(\mathrm{S})\end{array}$ \\
\hline 174 & 1235 & 2874 & 19,49 & $\mathrm{v}$ C-C $(\mathrm{S}$ and $\mathrm{A})$ \\
\hline 175 & 1239 & 244 & 23,19 & \multirow[t]{2}{*}{ v C-C-C (S) } \\
\hline 176 & 1239 & 212 & 33,58 & \\
\hline 177 & 1240 & 429 & 12,34 & \multirow[t]{2}{*}{$\tau \mathrm{C}-\mathrm{H}$ in $\mathrm{CH}_{2}$ groups $(\mathrm{S})$} \\
\hline 178 & 1243 & 426 & 3,22 & \\
\hline 179 & 1257 & 4012 & 39,49 & v C-C (A) \\
\hline 180 & 1264 & 181 & 0,34 & $\tau \mathrm{C}-\mathrm{H}$ in $\mathrm{CH}_{2}$ groups $(\mathrm{S})$ \\
\hline 181 & 1266 & 37 & 0,48 & $\mathrm{v} \mathrm{C}=\mathrm{C}(\mathrm{R})$ \\
\hline 182 & 1271 & 248 & 6,46 & \multirow[t]{2}{*}{$\tau \mathrm{C}-\mathrm{H}$ in $\mathrm{CH}_{2}$ groups $(\mathrm{S})$} \\
\hline 183 & 1271 & 481 & 0,89 & \\
\hline
\end{tabular}




\begin{tabular}{|c|c|c|c|c|}
\hline 184 & 1276 & 134 & 1,41 & \\
\hline 185 & 1277 & 166 & 3,71 & \\
\hline 186 & 1284 & 162 & 0,43 & $\delta \mathrm{C}-\mathrm{H}(\mathrm{R})$ \\
\hline 187 & 1286 & 70 & 10,68 & \multirow{15}{*}{$\tau \mathrm{C}-\mathrm{H}$ in $\mathrm{CH}_{2}$ groups $(\mathrm{S})$} \\
\hline 188 & 1287 & 29 & 2,78 & \\
\hline 189 & 1288 & 265 & 2,42 & \\
\hline 190 & 1289 & 63 & 8,72 & \\
\hline 191 & 1293 & 153 & 1,47 & \\
\hline 192 & 1296 & 197 & 5,51 & \\
\hline 193 & 1305 & 205 & 5,85 & \\
\hline 194 & 1307 & 208 & 1,09 & \\
\hline 195 & 1320 & 186 & 22,10 & \\
\hline 196 & 1320 & 125 & 13,15 & \\
\hline 197 & 1322 & 74 & 11,24 & \\
\hline 198 & 1322 & 145 & 13,05 & \\
\hline 199 & 1323 & 181 & 10,18 & \\
\hline 200 & 1323 & 171 & 2,55 & \\
\hline 201 & 1327 & 270 & 110,08 & \\
\hline 202 & 1334 & 160 & 3,97 & \multirow[t]{15}{*}{$\zeta \mathrm{C}-\mathrm{H}$ in $\mathrm{CH}_{2}$ groups $(\mathrm{S})$} \\
\hline 203 & 1336 & 107 & 6,02 & \\
\hline 204 & 1338 & 280 & 4,77 & \\
\hline 205 & 1339 & 313 & 6,55 & \\
\hline 206 & 1340 & 98 & 22,11 & \\
\hline 207 & 1341 & 124 & 5,79 & \\
\hline 208 & 1343 & 100 & 1,29 & \\
\hline 209 & 1344 & 193 & 10,91 & \\
\hline 210 & 1344 & 104 & 15,24 & \\
\hline 211 & 1348 & 109 & 2,74 & \\
\hline 212 & 1349 & 41 & 14,44 & \\
\hline 213 & 1350 & 18 & 2,84 & \\
\hline 214 & 1352 & 81 & 50,27 & \\
\hline 215 & 1358 & 76 & 6,62 & \\
\hline 216 & 1360 & 65 & 1,44 & \\
\hline 217 & 1379 & 212 & 9,20 & $\delta \mathrm{C}-\mathrm{H}$ in $\mathrm{CH}_{3}$ and $\mathrm{CH}$ groups $(\mathrm{S})$ \\
\hline 218 & 1380 & 112 & 2,76 & $\delta \mathrm{C}-\mathrm{H}$ in $\mathrm{CH}_{3}$ groups $(\mathrm{S})$ \\
\hline 219 & 1384 & 310 & 18,14 & $\delta \mathrm{C}-\mathrm{H}$ in $\mathrm{CH}_{3}$ and $\mathrm{CH}$ groups $(\mathrm{S})$ \\
\hline 220 & 1387 & 122 & 7,53 & $\delta \mathrm{C}-\mathrm{H}$ in $\mathrm{CH}_{3}$ groups $(\mathrm{S})$ \\
\hline 221 & 1395 & 16 & 4,03 & $\mathrm{v} \mathrm{C}=\mathrm{C}(\mathrm{R})$ \\
\hline 222 & 1435 & 305 & 5,17 & \multirow[t]{9}{*}{$\delta \mathrm{C}-\mathrm{H}$ in $\mathrm{CH}_{2}$ groups (S) } \\
\hline 223 & 1436 & 309 & 4,88 & \\
\hline 224 & 1446 & 203 & 8,91 & \\
\hline 225 & 1446 & 214 & 8,81 & \\
\hline 226 & 1452 & 443 & 0,04 & \\
\hline 227 & 1454 & 415 & 0,28 & \\
\hline 228 & 1460 & 190 & 2,39 & \\
\hline 229 & 1461 & 158 & 4,98 & \\
\hline 230 & 1465 & 262 & 1,79 & \\
\hline
\end{tabular}




\begin{tabular}{|c|c|c|c|c|}
\hline 231 & 1465 & 149 & 1,81 & \\
\hline 232 & 1465 & 111 & 2,14 & \\
\hline 233 & 1466 & 131 & 1,80 & \\
\hline 234 & 1466 & 37 & 0,26 & \multirow[t]{6}{*}{$\delta \mathrm{C}-\mathrm{H}$ in $\mathrm{CH}_{3}$ and $\mathrm{CH}_{2}$ groups (S) } \\
\hline 235 & 1468 & 158 & 2,48 & \\
\hline 236 & 1476 & 46 & 9,21 & \\
\hline 237 & 1478 & 334 & 4,92 & \\
\hline 238 & 1480 & 134 & 10,65 & \\
\hline 239 & 1480 & 392 & 4,96 & \\
\hline 240 & 1482 & 164 & 3,76 & \multirow[t]{2}{*}{$\delta \mathrm{C}-\mathrm{H}$ in $\mathrm{CH}_{2}$ groups $(\mathrm{S})$} \\
\hline 241 & 1483 & 96 & 1,95 & \\
\hline 242 & 1489 & 59 & 0,59 & $8 \mathrm{C}-\mathrm{H}$ in $\mathrm{CH}_{3}$ and $\mathrm{CH}_{2}$ groups (S) \\
\hline 243 & 1493 & 63 & 80,55 & $\mathrm{v} \mathrm{C}=\mathrm{C}(\mathrm{R})$ \\
\hline 244 & 1494 & 99 & 1,06 & $\delta \mathrm{C}-\mathrm{H}$ in $\mathrm{CH}_{3}$ and $\mathrm{CH}_{2}$ groups $(\mathrm{S})$ \\
\hline 245 & 1523 & 91 & 0,07 & \multirow[t]{2}{*}{$\mathrm{v} \mathrm{C}=\mathrm{C}(\mathrm{R})$} \\
\hline 246 & 1597 & 51796 & 3,19 & \\
\hline 247 & 1622 & 1301 & 46,88 & \multirow[t]{2}{*}{ v $\mathrm{C}=\mathrm{C}(\mathrm{S})$} \\
\hline 248 & 1622 & 1679 & 56,22 & \\
\hline 249 & 1709 & 578 & 128,67 & \multirow[t]{2}{*}{$\mathrm{v} C=\mathrm{O}(\mathrm{S})$} \\
\hline 250 & 1710 & 1581 & 479,47 & \\
\hline 251 & 2228 & 43780 & 6,65 & \multirow[t]{2}{*}{$\mathrm{v} C \equiv \mathrm{C}(\mathrm{A})$} \\
\hline 252 & 2244 & 21633 & 1,06 & \\
\hline 253 & 2845 & 131 & 9,11 & \multirow[t]{6}{*}{$v \mathrm{C}-\mathrm{H}$ in $\mathrm{CH}$ group $(\mathrm{S})$} \\
\hline 254 & 2846 & 136 & 8,45 & \\
\hline 255 & 2869 & 27 & 4,01 & \\
\hline 256 & 2870 & 66 & 2,61 & \\
\hline 257 & 2876 & 207 & 8,23 & \\
\hline 258 & 2879 & 75 & 0,93 & \\
\hline 259 & 2883 & 347 & 9,13 & \multirow[t]{19}{*}{$v \mathrm{C}-\mathrm{H}$ in $\mathrm{CH}_{2}$ group $(\mathrm{S})$} \\
\hline 260 & 2884 & 423 & 13,16 & \\
\hline 261 & 2892 & 24 & 44,70 & \\
\hline 262 & 2892 & 11 & 36,04 & \\
\hline 263 & 2906 & 301 & 9,07 & \\
\hline 264 & 2906 & 315 & 10,01 & \\
\hline 265 & 2912 & 166 & 22,04 & \\
\hline 266 & 2915 & 212 & 1,37 & \\
\hline 267 & 2916 & 19 & 58,31 & \\
\hline 268 & 2916 & 49 & 43,48 & \\
\hline 269 & 2920 & 37 & 49,23 & \\
\hline 270 & 2921 & 48 & 36,64 & \\
\hline 271 & 2921 & 61 & 23,46 & \\
\hline 272 & 2934 & 73 & 21,05 & \\
\hline 273 & 2936 & 105 & 45,98 & \\
\hline 274 & 2939 & 384 & 5,07 & \\
\hline 275 & 2940 & 209 & 37,31 & \\
\hline 276 & 2940 & 63 & 32,85 & \\
\hline 277 & 2944 & 375 & 18,51 & \\
\hline
\end{tabular}




\begin{tabular}{|c|c|c|c|c|}
\hline 278 & 2947 & 517 & 60,99 & \\
\hline 279 & 2948 & 352 & 40,68 & \\
\hline 280 & 2961 & 385 & 18,36 & \\
\hline 281 & 2963 & 213 & 50,84 & \\
\hline 282 & 2964 & 271 & 42,01 & \\
\hline 283 & 2968 & 70 & 36,36 & \\
\hline 284 & 2969 & 148 & 32,39 & \\
\hline 285 & 2969 & 245 & 34,01 & \\
\hline 286 & 2969 & 128 & 23,82 & \\
\hline 287 & 2972 & 130 & 35,76 & \\
\hline 288 & 2975 & 163 & 40,77 & \\
\hline 289 & 2980 & 219 & 24,53 & \\
\hline 290 & 2981 & 219 & 38,66 & \\
\hline 291 & 2981 & 34 & 25,84 & \\
\hline 292 & 2982 & 33 & 31,36 & \\
\hline 293 & 2983 & 186 & 29,25 & \\
\hline 294 & 2984 & 145 & 26,08 & \\
\hline 295 & 2992 & 267 & 22,23 & \\
\hline 296 & 2992 & 263 & 19,59 & \\
\hline 297 & 2993 & 144 & 44,09 & \multirow[t]{2}{*}{$v \mathrm{C}-\mathrm{H}$ in $\mathrm{CH}_{3}$ group $(\mathrm{S})$} \\
\hline 298 & 2998 & 185 & 30,87 & \\
\hline 299 & 3006 & 228 & 43,45 & \multirow[t]{2}{*}{$v \mathrm{C}-\mathrm{H}$ in $\mathrm{CH}_{2}$ group (S) } \\
\hline 300 & 3009 & 178 & 35,13 & \\
\hline 301 & 3022 & 51 & 23,16 & \multirow[t]{2}{*}{$v \mathrm{C}-\mathrm{H}$ in $\mathrm{CH}_{3}$ group $(\mathrm{S})$} \\
\hline 302 & 3041 & 62 & 14,29 & \\
\hline 303 & 3057 & 221 & 12,12 & \multirow[t]{2}{*}{$v$ C-H (S) } \\
\hline 304 & 3057 & 216 & 12,00 & \\
\hline 305 & 3079 & 42 & 12,32 & \multirow[t]{3}{*}{$v$ C-Hasym. (R) } \\
\hline 306 & 3080 & 151 & 3,22 & \\
\hline 307 & 3094 & 21 & 14,63 & \\
\hline 308 & 3097 & 319 & 1,44 & v C-Hsym. (R) \\
\hline 309 & 3592 & 164 & 16,30 & \multirow[t]{2}{*}{$v \mathrm{O}-\mathrm{H}(\mathrm{S})$} \\
\hline 310 & 3603 & 266 & 8,86 & \\
\hline
\end{tabular}

Abbreviations: $\mathrm{S}$ - stator, $\mathrm{R}$ - rotator, A - axle, $v$ - stretching, $\delta$ - bending, $\gamma$ - out of plane bending, $\zeta$ wagging, $\tau-$ twisting, sym. - symmetric, asym. - asymmetric. 


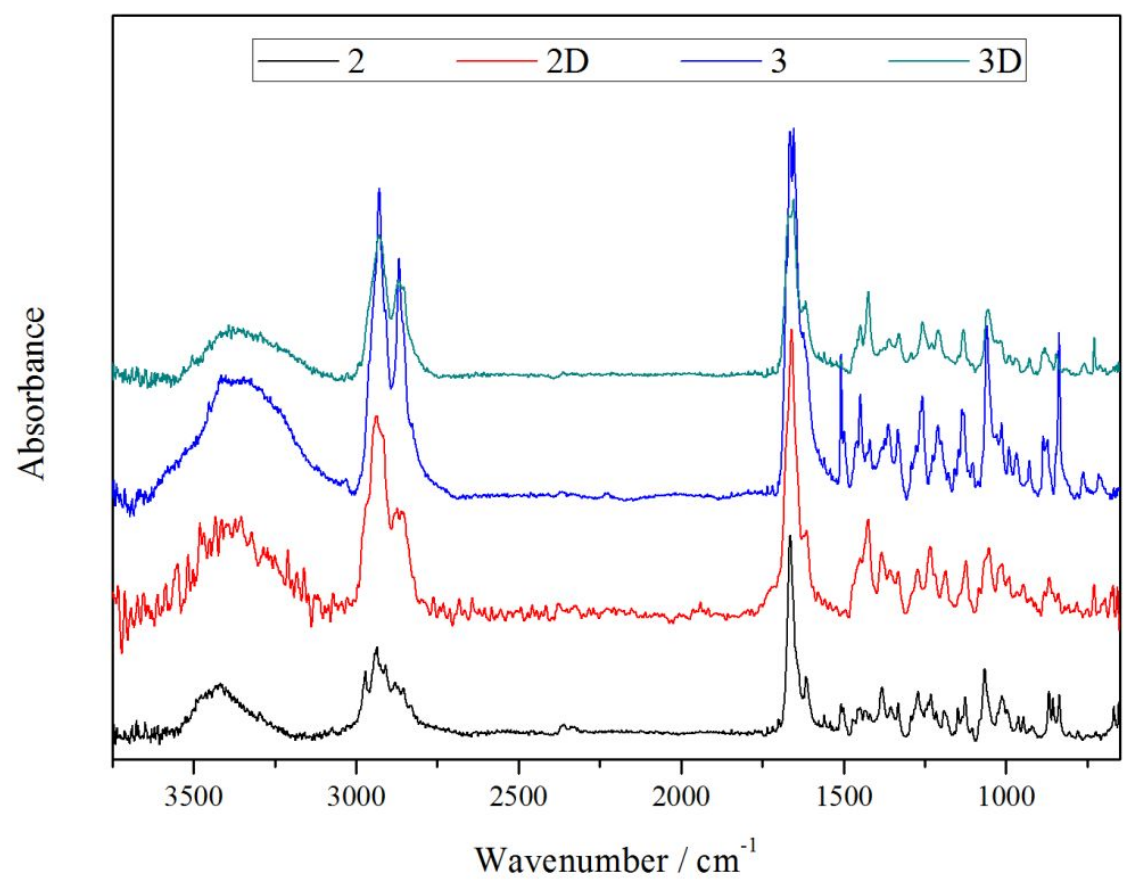

Figure S1. ATR-IR spectra of rotors 2, 2D, 3, 3D. 

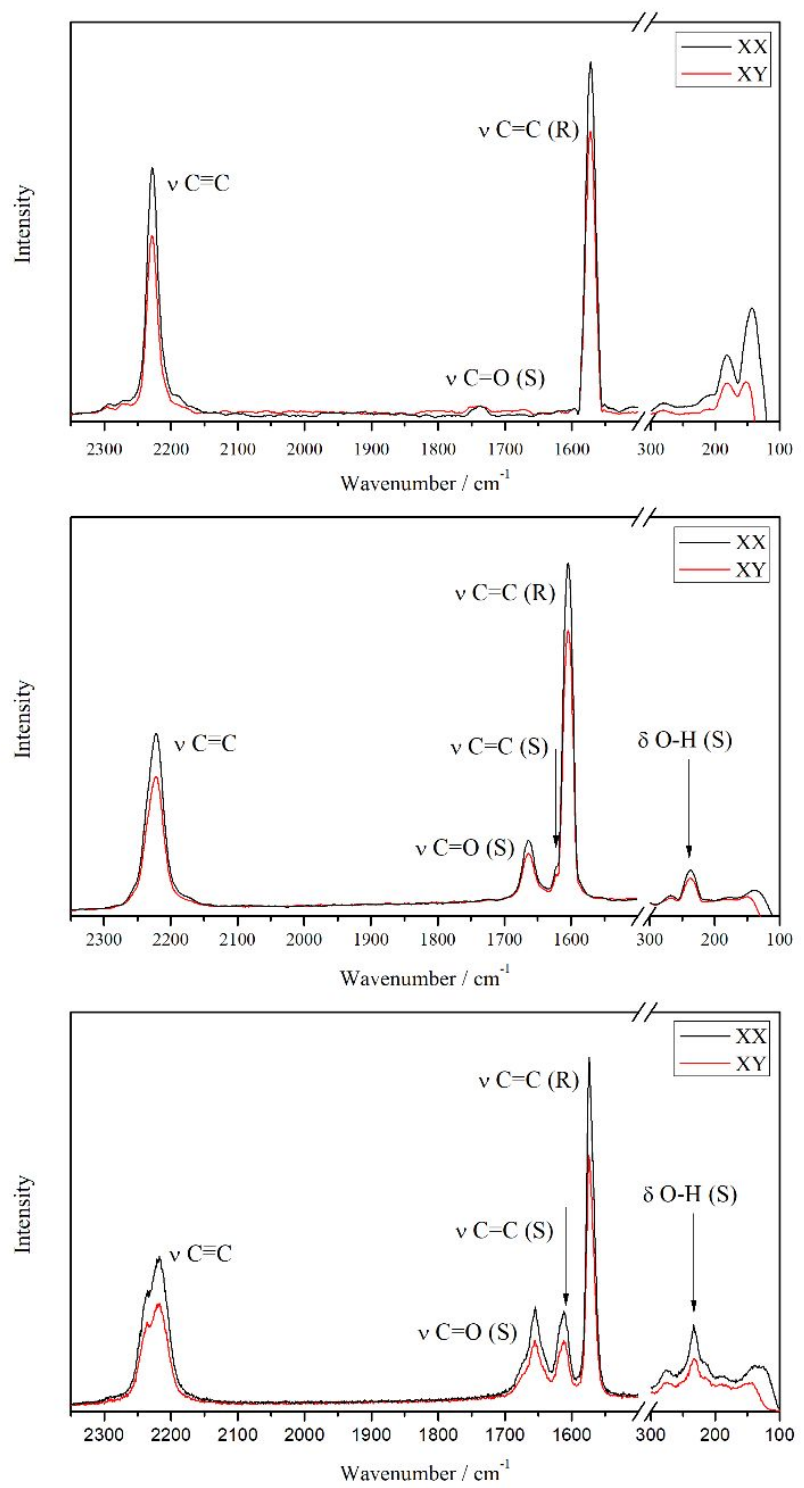

Figure S2. Polarized spectra of rotors 1D (a), 2 (b) and 2D (c). 

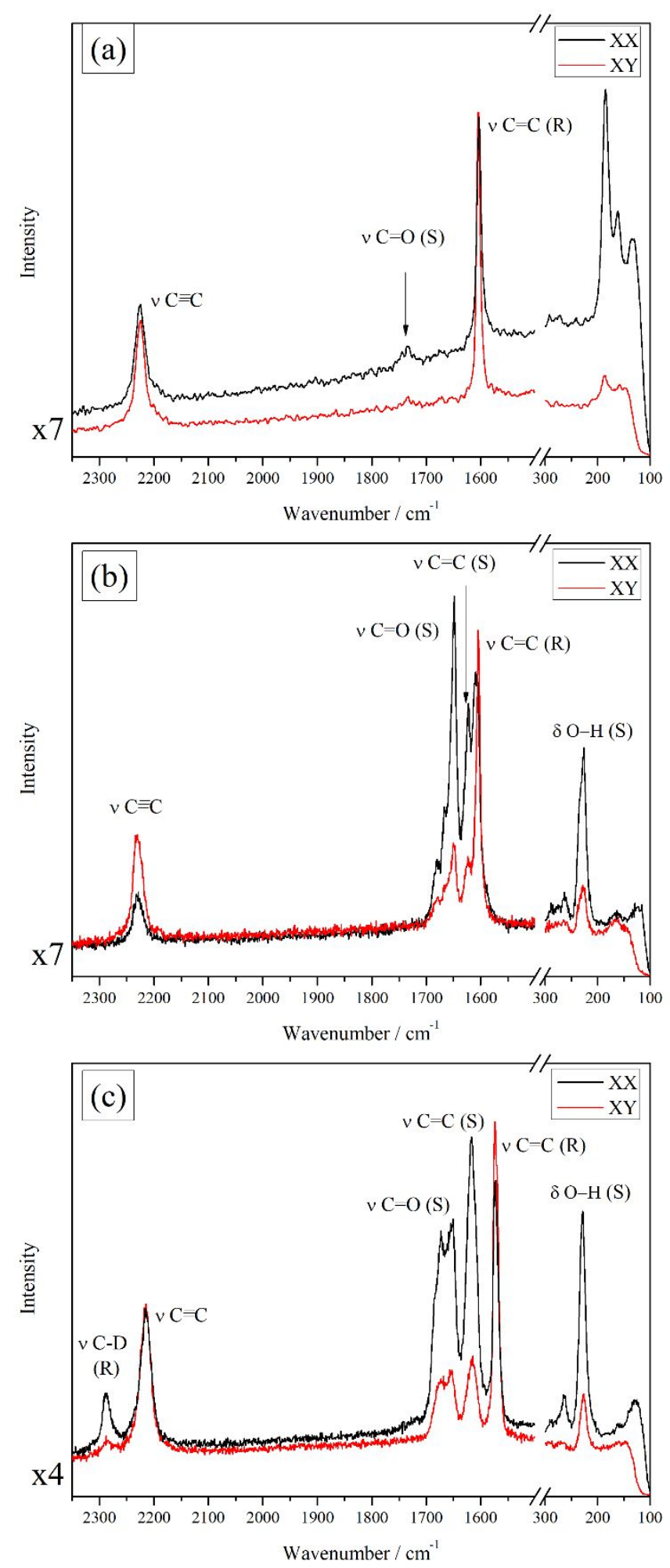

Figure S3. Polarized Raman spectra of rotors 1 (a), 3 (b) and 3D (c) after rotating the sample by $90^{\circ}$ (the number in the bottom of each figure signifies how many times the intensity was multiplied in relation to Figure 7). 


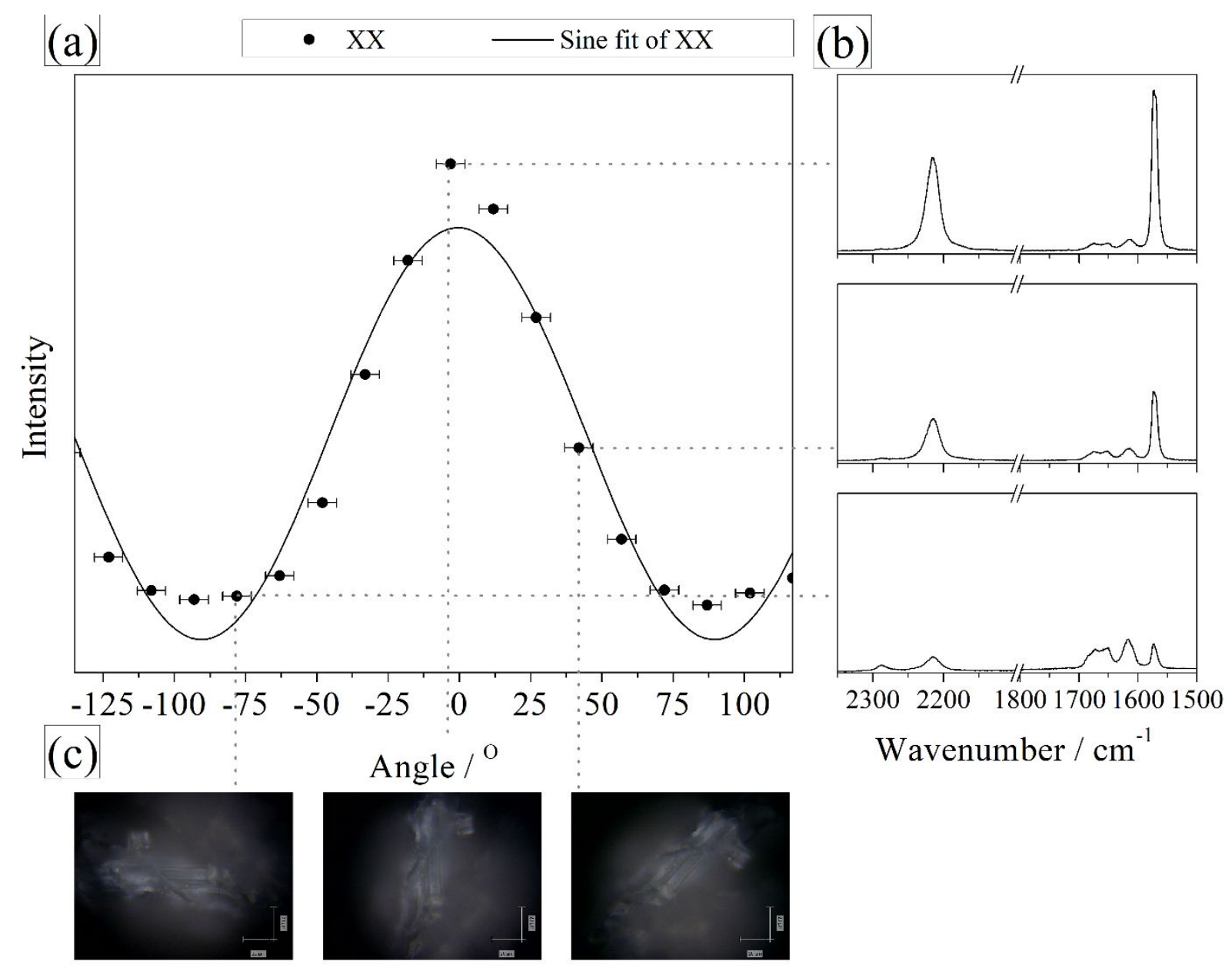

Figure S4. The dependencies of intensity vs. angle of rotation of the sample for the band assigned to stretching vibration of $\mathrm{C}=\mathrm{C}$ bonds in the rotator recorded in parallel (XX) polarization for rotor 3D. The Raman spectra recorded for corresponding angles are depicted on the right side. 


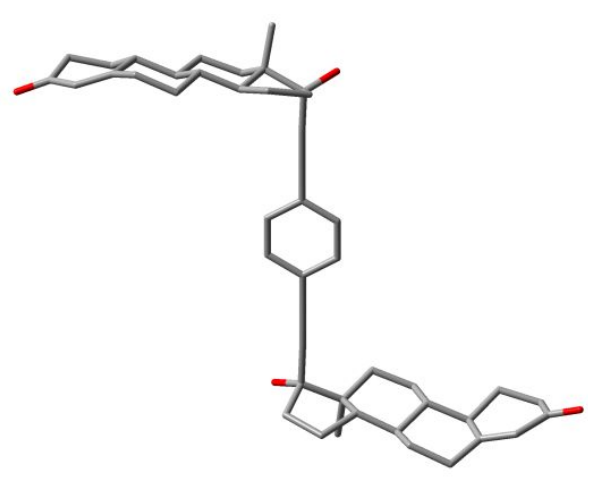

0 kcal/mol

$17.75 \%$

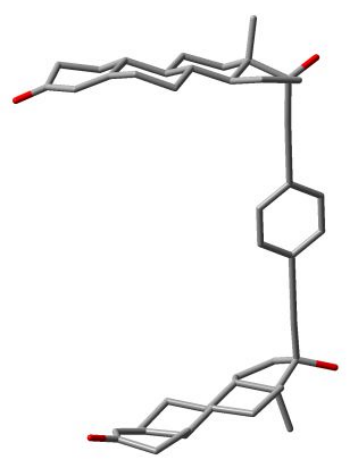

$0.17 \mathrm{kcal} / \mathrm{mol}$

$13.40 \%$

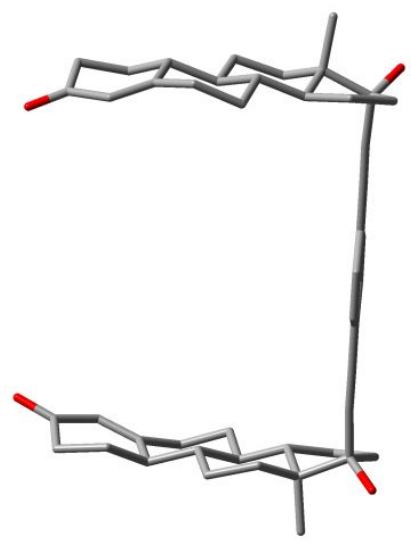

$0.34 \mathrm{kcal} / \mathrm{mol}$

$9.98 \%$

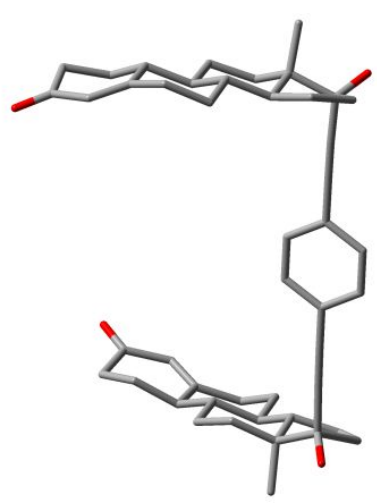

$0.02 \mathrm{kcal} / \mathrm{mol}$

$17.10 \%$

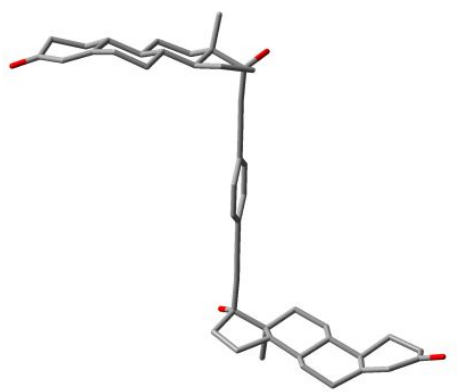

$0.22 \mathrm{kcal} / \mathrm{mol}$

$12.16 \%$

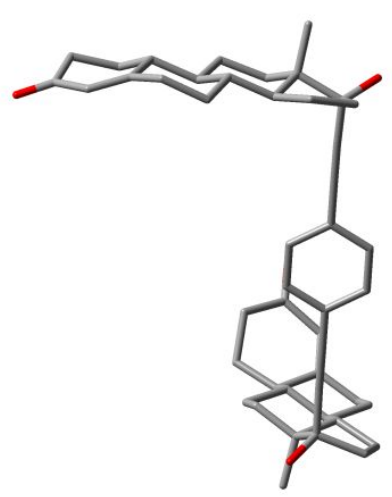

$1.22 \mathrm{kcal} / \mathrm{mol}$

$2.26 \%$

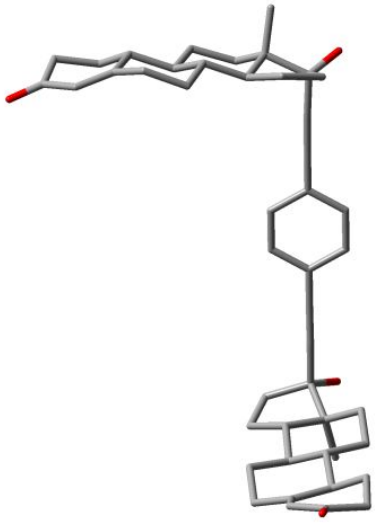

$0.1 \mathrm{kcal} / \mathrm{mol}$

$14.97 \%$

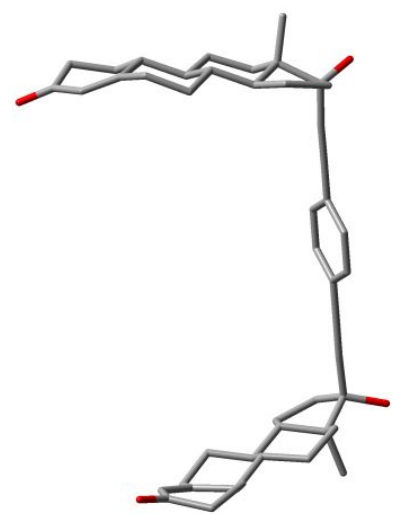

$0.31 \mathrm{kcal} / \mathrm{mol}$

$10.56 \%$

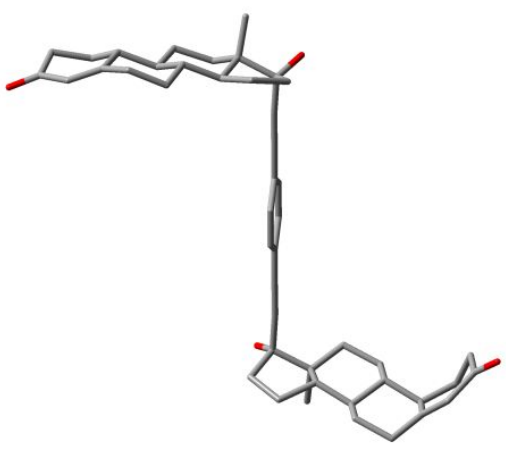

$1.35 \mathrm{kcal} / \mathrm{mol}$

$1.82 \%$

Figure S5. An overview of conformational search for compounds 3 carried out at B3LYP/6$31 G(d)$ level of theory (calculations were performed for the structures with methyl group at C13). 

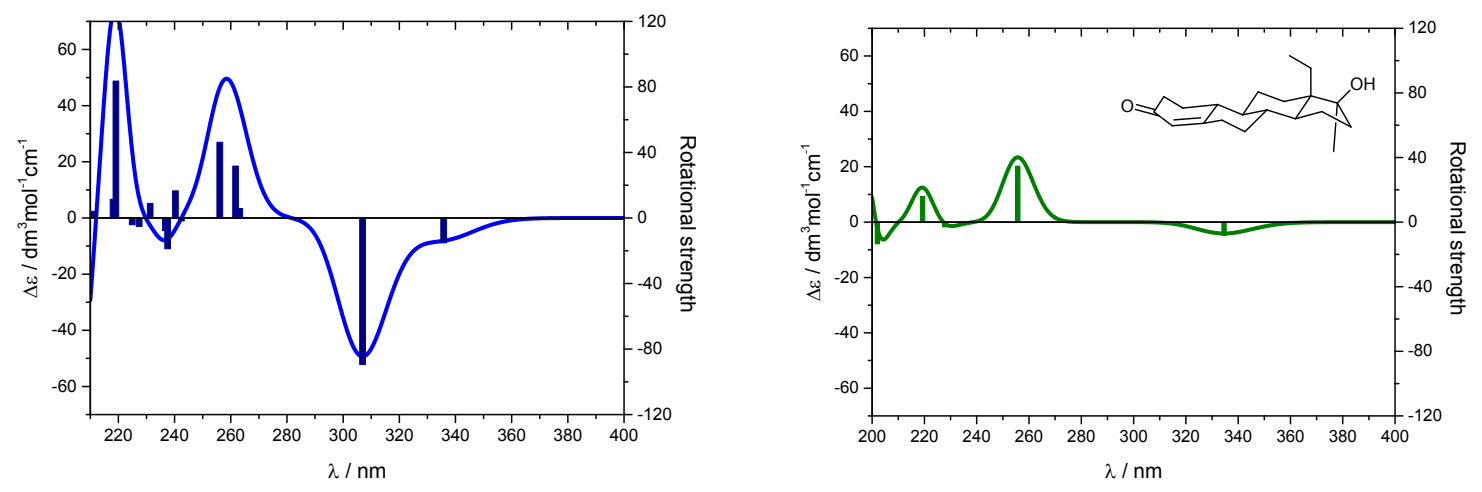

Figure S6. Simulated spectrum of 3a (left) and steroidal unit (b) at CAM-B3LYP/def2$\mathrm{TZVP} / \mathrm{PCM}\left(\mathrm{CH}_{3} \mathrm{CN}\right)$ level of theory (band width $=0.2 \mathrm{eV}, \mathrm{UV}$ shift $=30 \mathrm{~nm}$ ). 